EPJ manuscript No.

2(will be inserted by the-editoll)uang: Self-organized model for information spread in financial markets

\title{
Self-organized model for information spread in financial markets
}

\author{
Zhi-Feng Huang \\ Institute for Theoretical Physics, Cologne University, D-50923, Köln, Germany
}

\begin{abstract}
A self-organized model with social percolation process is proposed to describe the propagations of information for different trading ways across a social system and the automatic formation of various groups within market traders. Based on the market structure of this model, some stylized observations of real market can be reproduced, including the slow decay of volatility correlations, and the fat tail distribution of price returns which is found to cross over to an exponential-type asymptotic decay in different dimensional systems.
\end{abstract}

PACS. 89.90. $+\mathrm{n}$ Other topics of general interest to physicists - 87.23.Ge Dynamics of social systems 05.45.Tp Time series analysis - 64.60.Cn Order-disorder transformations; statistical mechanics of model systems

Recently, microscopic models for financial markets have

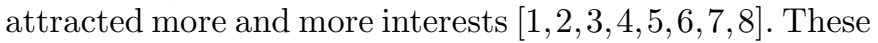
models are based on the empirical findings of high-frequency market data, such as the fat tails in the distribution of price changes, the fast decaying of linear correlation, as well as the existence of long-term volatility correlations [2, 3, 4, 9], and the intrinsic structure of financial markets, including the mutual interactions among market participants through herding and imitation behaviors [6,7] or switching from one strategy group to another [8].

Although different mechanisms are used in these microscopic models to simulate the price formation processes, some of the stylized observations of real markets can be reproduced. In one set of these models, the Cond-Bouchaud herding model [6] and the related percolation models 10 , 11, the power-law asymptotic behavior in the tail of price return has been obtained, with an exponent well outside the stable Lévy regime [1] as found in real data [12, 13]. Recently, this Cond-Bouchaud percolation model has been modified by introducing a feedback mechanism between price return $R$ and trader activity $a: a \rightarrow a+\alpha R$, where $\alpha$ is the factor representing the sensitivity to price fluctuations [11]. Then the volatility clustering can be produced, and more interestingly, the empirically observed asymmetric pattern of sharp peaks and flat troughs in the market prices [14] can be incorporated.

In the percolation-type model [6, 10, 11], investors are randomly distributed on a lattice with certain concentration and through the neighboring connections they form the percolation clusters, corresponding to herding groups or companies with probability $a$ of trading (activity) which is set to be the same for all groups. However, the concentration of investors on the lattice is to be pre-setted for obtaining resonable results. Here we introduce a self-

\footnotetext{
* E-mail address: zfh@thp.uni-koeln.de
}

organized model for information spread based on the social percolation process [15, 16], where the investor groups with various trading activities and sizes are formed automatically as a result of the propagations of trading information across a social network.

Consider a $d$ dimensional lattice representing a social system with each site located by a trader, that is, the whole lattice is occupied. When participating in the market each trader obeys a certain trading way, which can be simply represented by the trading activity measuring the frequency of putting the buy or sell orders, and she may change her behavior due to the influence of outside information about trading ways if she believes that the information is attractive enough and the new trading way can lead to more profits. Suppose that the information of various trading ways is not public, and spreads among traders according to the communication structure of the social system, which for simplification, is considered as the transfer along nearest-neighbor links of lattice. Thus, a higher dimensional lattice corresponds to the society with more connections among agents [16]. All the traders accepting the same information form a cluster or group and will exhibit the same behavior in the market as they obey the same trading way.

First, we study the process of information spread for different trading ways, characterized simply by activity $a(\tau)$ at each spread step $\tau$ (and the sensitivity to price change $\alpha(\tau)$ if considering the feedback effect as described above [11]). In the way of larger $a(\tau)$ the trading will be made more frequently. Each information of trading way has an attractiveness $q(\tau)$, related to trader's personal opinion about its profitability. The dynamics of the model is:

- At one step $\tau$, a new information of trading way with attractiveness $q(\tau)$ and activity $a(\tau)$ both determined 
randomly appears and starts at few informed sites to spread by nearest-neighbor connections among the traderst $p_{i}$ are set between 0 and 1 and $\delta p$ shown below is a small fixed parameter).

- Each step $\tau$ consists of consecutive substeps of the spread between traders: After receiving the information from one of her neighbors at one substep, trader $i$ will accept it and obey this new trading way if its attractiveness is larger than her personal preference, i.e., $q(\tau)>p_{i}$. Then she will spread the information further to the other neighbors, and increase her preference by $\delta p$, i.e., $p_{i} \rightarrow p_{i}+\delta p$, as it should be more difficult and needs larger attractiveness for someone to change her mind at the next step $\tau+1$ to another new way. Otherwise, the trader $i$ will keep the old state, including the old trading way and preference, and will not spread the message to her neighbors until the end of this step $\tau$. Then, the next substep begins with the similar judgement and spread procedure.

- The spread of one step $\tau$ will continue until there is no source of information for propagation and the procedure stops by itself, that is, all the neighbors of all the traders accepting the information have already received it, and a trader group or cluster with members accepting the common information is formed. Then the next step $\tau+1$ starts with the incoming of another new information of attractiveness $q(\tau+1)$ and activity $a(\tau+1)$, also selected randomly between 0 and 1 , and has the spread procedure similar to above. In particular, for a trader $i$ belonging to a cluster of previous information, when she receives the new information with $q(\tau+1)>p_{i}$ she will accept it and change her trading way to the new one. Consequently, the size of that old cluster will decrease by 1 with the expansion of a new group. Otherwise, the trader will still remain in the old cluster. Thus, if the new information is attractive enough, the spread of it will form a new group which may invade the old ones or even make some of them disappearing. Up to now, all traders within the same cluster share the same activity $a(\tau)$ which was valid when that cluster was formed.

Compared with the social percolation model [15, 16], here there is no feedback on the attractiveness $q$ of information, instead, new information with randomly selected attractiveness appears at each new step. Moreover, the preferences of investors not accepting the new information or uninformed are not changed, instead of decreasing, and then the trader preferences increase with the acceptance of more and more information. After a large number of steps for the spread of different trading information, the trader preferences of the system are close to the upper limit 1 and the newly appearing information has little influence on the trader structure. Thus, through a self-organized process the market traders in this social system automatically form groups or clusters of various sizes and activities due to the acceptance of different trading information, and these clusters may correspond to the herding groups of investors who imitate each other or individual groups with members using the same trading activity $a$ and acting in rsthe same manner. Here the groups or clusters with larger size correspond to the agents in the real market who have bigger influence, and different activities of groups represent the phenomenon of the real market that the agents have different trading timescale and some of them trade more frequently than the other.

Fig. 1 shows the results of our simulations for different hypercubic lattices with length $L$ and dimension $d$, where $n_{s}$ denotes the average number of clusters containing $s$ sites, and only two parameters are to be chosen: $\delta p=0.001$ and $\tau=10^{5}$ steps. Initially the trader preferences $p_{i}$ are distributed randomly between 0 and 1 , and each step starts with one randomly selected site on one boundary. Thus, the sites close to this boundary represent the more informed traders in the market. A Leath-type algorithm [17] is used for the spread procedures across the lattices with helical boundary conditions. From Fig. 1 a power law behavior similar to the percolation structure is obtained, but only for small and intermediate sizes of clusters. Compared with percolation theory 18, the effective power law exponent in the present model is smaller and can be lower than 2 in low dimensions with a finite value of $\sum s n_{s}$ which is very close to 1 . This phenomenon for the slower decaying of $n_{s}$ may be attributed to the much heavier tail in the distribution of cluster sizes in present model. In the percolation lattice a cluster with size roughly comparable to the total system size (largest or "infinite" percolating cluster) appears at or above the percolation threshold, while in the model here an earlier percolating cluster may be invaded by new-coming information that is attractive enough compared with traders' preferences, and then its size is diminished with the formation of more and smaller clusters. Thus, in the present model the sites belonging to the same cluster need not be nearest-neighbors to each other as in percolation models 10. For large $s$, it is not clear whether an asymptotic stretched exponential behavior similar to percolation structure above the critical threshold exists in present model, and more detailed work is needed.

Next, we study the trading behaviors of these market groups or clusters that are determined after enough steps of the spreading process as described above. For simplicity, during the trading and price formation processes simulated below, the influence of new-coming information, i.e., the change of market structure for trader groups, is not considered. The trading of each group $j$ is represented by an activity $a_{j}$ obtained from different information in the spread process and randomly distributed between two limits $a_{\max }$ and $a_{\min }$, and then at each time step, group $j$ decides to make orders with probability $a_{j}$ (with equal probability $a_{j} / 2$ for buying or selling) or be inactive with probability $1-a_{j}$. This mechanism does not require that each buy order should match a sell order, and the balance can be made by assuming the involvement of market makers outside the model. Assume that all investors in one group contribute the same amount of trading orders, and then the trading amount of that group is proportional to the group size. Moreover, the price return $R$ at each time 
step $t$ (used for trading process and different from the spread step $\tau$ ), which is defined as the change over time interval $\Delta t$ of logarithm of price, is supposed to be proportional to the excess demand [6, 19], that is, the difference between demand and supply obtained from the sum over all active clusters, and normalized by the system size $L^{d}$, that is, $R \rightarrow R / L^{d}$, to avoid the influence of lattice size.

Figs. 2 and 3 give the results for the probability distribution of price returns on the simple cubic lattice, and similar behaviors are found for other dimensions from two to seven. In the simulations, the unit of time increment $\Delta t$ is to be chosen appropriately in order to avoid the fluctuations due to random decisions, and here we select 5 time steps as the unit interval $\Delta t=1$. Fig. 2 shows a semi-log plot of the probability distribution of normalized returns, defined as $r=(R-\langle R\rangle) / \sigma$ with the average of return $\langle R\rangle$ (about 0 ) over the time series and the volatility $\sigma=\left(\left\langle R^{2}\right\rangle-\langle R\rangle^{2}\right)^{1 / 2}$, for $a_{\max }=0.02, a_{\min }=0.0001$, and different time scales $\Delta t=1,2,10$, and 100. With the increasing of time interval $\Delta t$, a crossover toward the Gaussian distribution is observed from the figure, in agreement with the finding of empirical financial data 13 .

In our simulations the asymptotic form of the fat tail distribution for small time scales is exponential-type, faster than the power law behavior with exponent about 4 found in recent empirical studies [12,13 and theoretical simulations [7, 8, 10]. Similar phenomena can be found in many real systems where the probability distributions often cross over to an exponential-type decay and exhibit curvature in the log-log plots after a limited range of scales for power law behavior, and a stretched exponential description has been shown to account well for many natural and economic distributions [20]. Very recently, an analytic form for the whole range of probability distribution and the corresponding Langevin equation have been derived 21 based on a theorem of general stationary random process and the Hong Kong Hang Seng Index data, and the asymptotic behavior for large price changes is an exponential-type decay: $P(r) \sim \exp (-\beta|r|) /|r|$. Fig. 3 is transformed from Fig. 2 by plotting $\ln |r| P(r)$ as a function of the absolute normalized return $|r|$ for the data of time interval $\Delta t=1$. Good fits to straight line in the tail region are found with $\beta=0.96 \pm 0.02$ (positive tail) and $0.96 \pm 0.03$ (negative tail) (fitted over well averaged region).

However, in above simulations the persistence of volatility correlation is absent, similar to the standard CondBouchaud model [6]. To reproduce the empirical facts of volatility clustering and asymmetry of bubbles and crashes in stock markets, we follow the method in 11 to introduce a feedback between price fluctuations and group behaviors at each time step: $a_{j}(t+1)=a_{j}(t)+\alpha_{j} R$, that is, the investors are encouraged by the price increasing with more tradings, and are more prudent when price decreases. We keep $a_{j}$ in the interval from $a_{\min }$ to $a_{\max }$. Furthermore, as the trading ways of the groups are different with different viewpoints on the price changes, in our simulations the parameters $\alpha_{j}$ are set randomly between two limits $\alpha_{\max }$ and $\alpha_{\text {min. }}$. Figs. 10 to show the results of simulations in two, three, and seven dimensions, where $\alpha_{\max }=0.1, \alpha_{\min }=0$, and the cutoffs of activity $a_{\max }=0.2$ and $a_{\min }=0.003$. To make the initial values of group activities irrelevant, the first $10^{6}$ time steps of the simulations are skipped.

The properties of fat tails in the probability distribution of normalized price returns $r$ are shown in Fig. 4, for different simulations of time interval $\Delta t=1$ in square, cubic, and $d=7$ hypercubic systems, respectively. The exponential-type tail behaviors can also be observed in the figure, and similarly we transformed Fig. 4 into Fig. 5 by plotting $\ln r P(r)$ vs normalized positive returns $r$, with the similar behaviors for negative ones. In the tail region good fits to straight line similar to that of the simulations without feedback effect are obtained, with parameter value $\beta \approx 1.1$ for different dimensional systems $\left(\beta=1.05 \pm 0.01\right.$ for size $1001^{2}, \beta=1.108 \pm 0.007$ for size $101^{3}$, and $\beta=1.109 \pm 0.007$ for size $7^{7}$, fitted over well averaged data). Simulations for other dimensions give the similar results.

The persistence of long-range volatility correlation, which is defined as the autocorrelation between the absolute value of price return, i.e., $[\langle|R(t)||R(t+T)|\rangle-\langle|R(t)|\rangle\langle| R(t+$ $T)|\rangle] /\left[\left\langle|R(t)|^{2}\right\rangle-\langle|R(t)|\rangle^{2}\right]$, is shown in Fig. 6 for 2, 3, and 7 dimensional lattices and time scale $\Delta t=1$, while the linear autocorrelation for the price returns is around zero. The inset of Fig. 6 gives the slow decay of volatility correlations in log-log plots, representing an asymptotic power-law with an exponent about 0.4 , similar to the empirical observation [3]. Volatility clustering as well as the asymmetry between sharp peaks and shallow valleys of the prices found empirically [14] are shown in Fig. 77, which gives the time series of one simulation on a $31^{3}$ simple cubic lattice.

Compared with the Cond-Bouchaud percolation models, where the system is fixed at (or slightly above) the percolation threshold or set at varying concentrations with results to be integrated over [10,11], the model here can automatically generate the market structure among traders, i.e., the trading groups or clusters, based on the social percolation process which is intrinsically different from the Cond-Bouchaud one, and the largest cluster need not be omitted for obtaining reasonable results. Moreover, in the present model the trading activity for different groups are different, reflecting the fact that in real market the agents have various timescales or frequencies of trading.

The qualitative results of the model here are robust with respect to parameters. The simulations with different parameters all lead to the same stylized features, including the properties of cluster size distribution after large enough steps of information spread, the fat tail of price return distribution described by exponential-type asymptotic decay, the long-range persistence of volatility autocorrelations, and the asymmetry between peaks and troughs of the prices. Different dimensional lattices simulate the different social societies with various degree of personal connections, and the dimensionality of the system does not influence the major results of the model. 
The author would like to thank Dietrich Stauffer and Sorin Solomon for very helpful discussions and comments. This work was supported by SFB 341.

\section{References}

1. G. J. Stigler, J. Business 37, 117 (1964).

2. J.-P. Bouchaud and M. Potters, Theory of Financial Risk (Cambridge University Press, Cambridge, 2000).

3. R. Mantegna and H. E. Stanley, An Introduction to Econophysics: Correlations and Complexity in Finance (Cambridge University Press, Cambridge, 1999).

4. J. D. Farmer, Computing in Science and Engineering 1, 26 (1999).

5. M. Levy, H. Levy, and S. Solomon, Economics Lett. 45, 103 (1994); J. Physique I 5, 1087 (1995).

6. R. Cont and J. P. Bouchaud, Macroeconomic Dynamics, in press, and e-print, cond-mat/9712318.

7. V. M. Eguiluz and M. G. Zimmermann, e-print, condmat/9908069.

8. T. Lux and M. Marchesi, Nature 297, 498 (1999).

9. R. N. Mantegna and H. E. Stanley, Nature 376, 46 (1995).

10. D. Stauffer and D. Sornette, Physica A 271, 496 (1999).

11. D. Stauffer and N. Jan, Physica A 277, 215 (2000).

12. T. Lux, Appl. Financial Economics 6, 463 (1996).

13. P. Gopikrishnan, V. Plerou, L. A. N. Amaral, M. Meyer, and H. E. Stanley, Phys. Rev. E 60, 5305 (1999).

14. B. M. Roehner and D. Sornette, Eur. Phys. J. B 4, 387 (1998).

15. S. Solomon, G. Weisbuch, L. de Arcangelis, N. Jan, and D. Stauffer, Physica A, 277,239 (2000).

16. Z. F. Huang, Int. J. Mod. Phys. C 11, 287 (2000).

17. H.G. Evertz, J. Stat. Phys. 70, 1075 (1993).

18. D. Stauffer and A. Aharony, Introduction to Percolation Theory (Taylor and Francis, London, 1994); M. Sahimi, Applications of Percolation Theory (Taylor and Francis, London, 1994).

19. J. D. Farmer, e-print, adapt-org/9812005.

20. J. Laherrère and D. Sornette, Eur. Phys. J. B. 2, 525 (1998).

21. L. H. Tang and Z. F. Huang, preprint. 


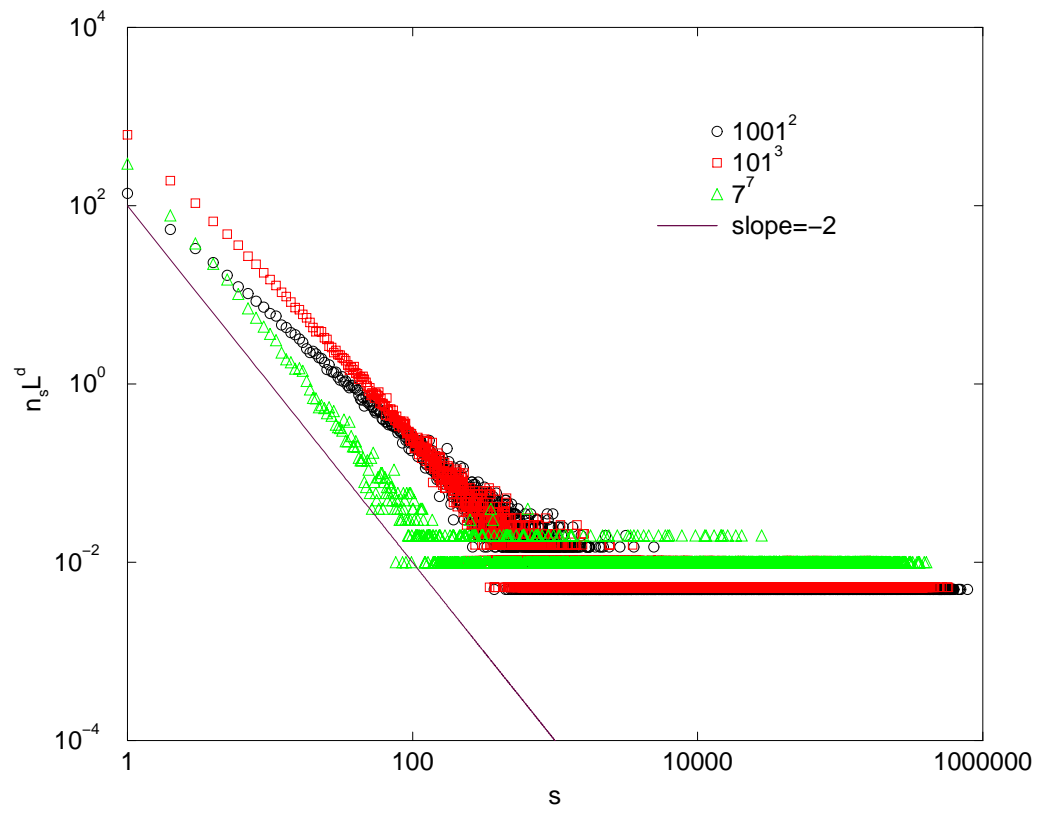

Fig. 1. Log-log plot of the cluster numbers containing $s$ sites after $\tau=10^{5}$ steps of the spread model with $\delta p=0.001$, for $1001^{2}$ square (averaged over 230 lattices), $101^{3}$ simple cubic (190 lattices), and $7^{7}$ hypercubic (100 lattices) systems. The slope of the straight line is -2 .

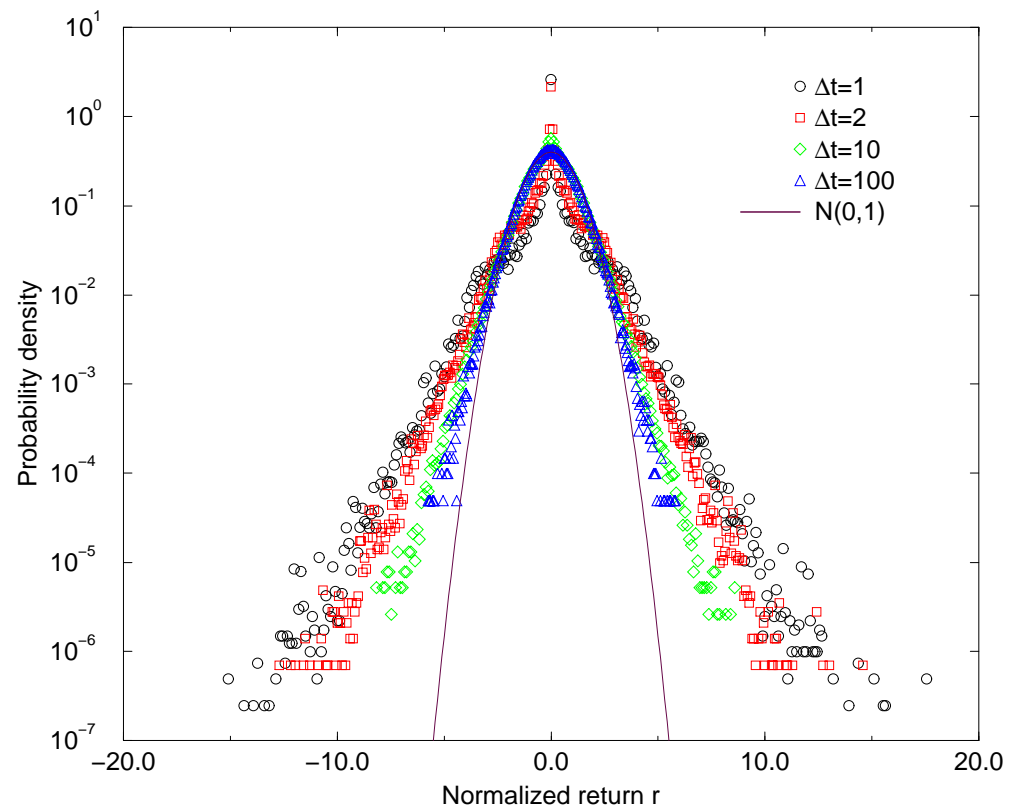

Fig. 2. Semi-log plot of the probability distribution of the normalized returns for different time intervals $\Delta t=1,2,10$, and 100 on $101^{3}$ cubic lattice with $a_{\max }=0.02$ and $a_{\mathrm{min}}=0.0001$ (averaged over 190 lattices). A crossover toward the Gaussian distribution (solid line) is shown with the increasing of time interval. 


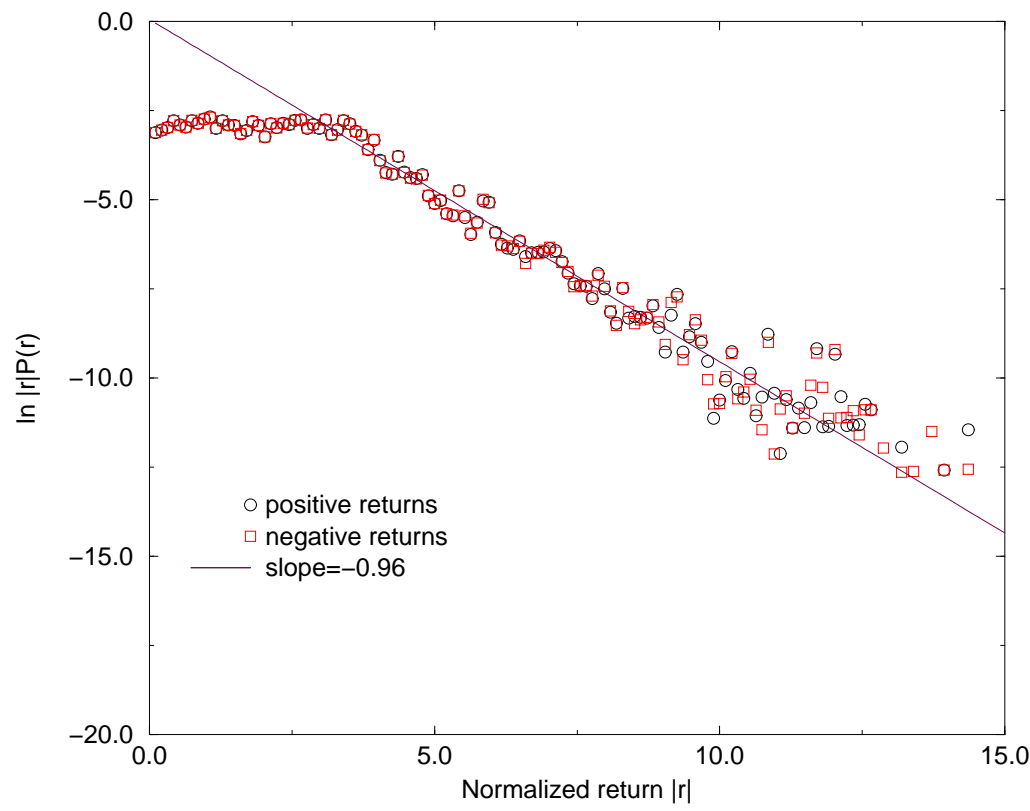

Fig. 3. Replot of Fig. 2 for time interval $\Delta t=1$ by showing $\ln |r| P(r)$ as a function of the absolute normalized return $|r|$, with a straight line of slope -0.96 .

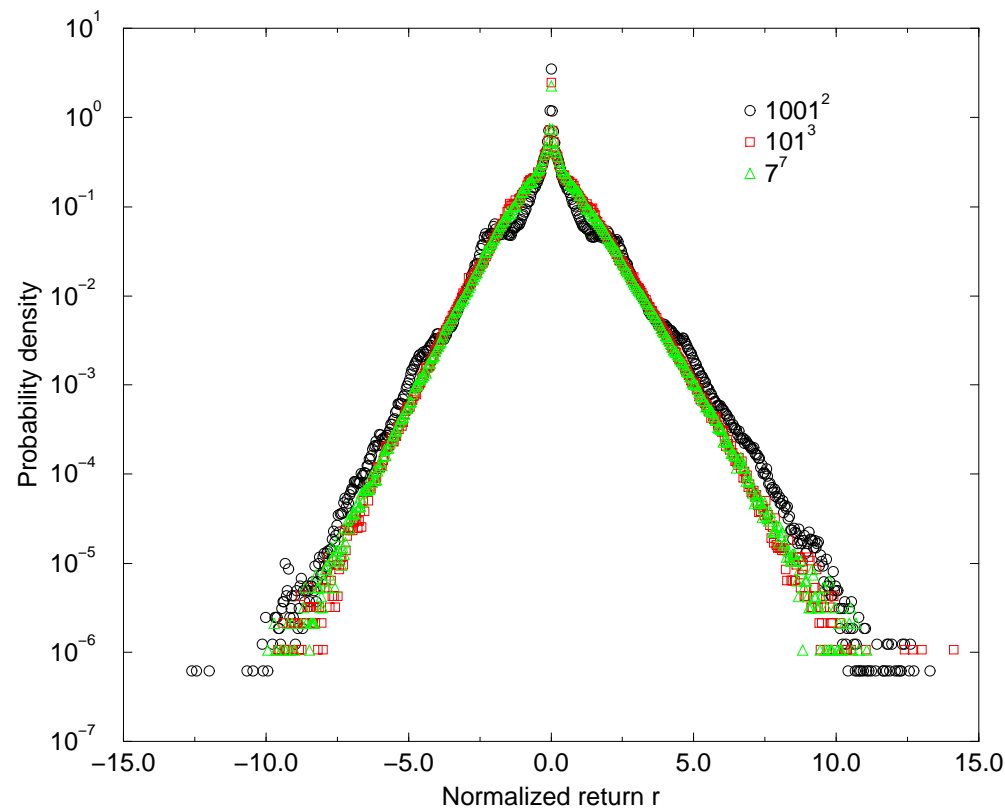

Fig. 4. Semi- $\log$ plot of the probability distribution of the normalized $\Delta t=1$ returns $r$ with $\alpha_{\max }=0.1, \alpha_{\min }=0, a_{\max }=0.2$, and $a_{\min }=0.003$, for $1001^{2}$ square (200 lattices), $101^{3}$ cubic (100 lattices), and $7^{7}$ hypercubic (100 lattices) systems. The feedback mechanism between price fluctuation and group activities is considered. 


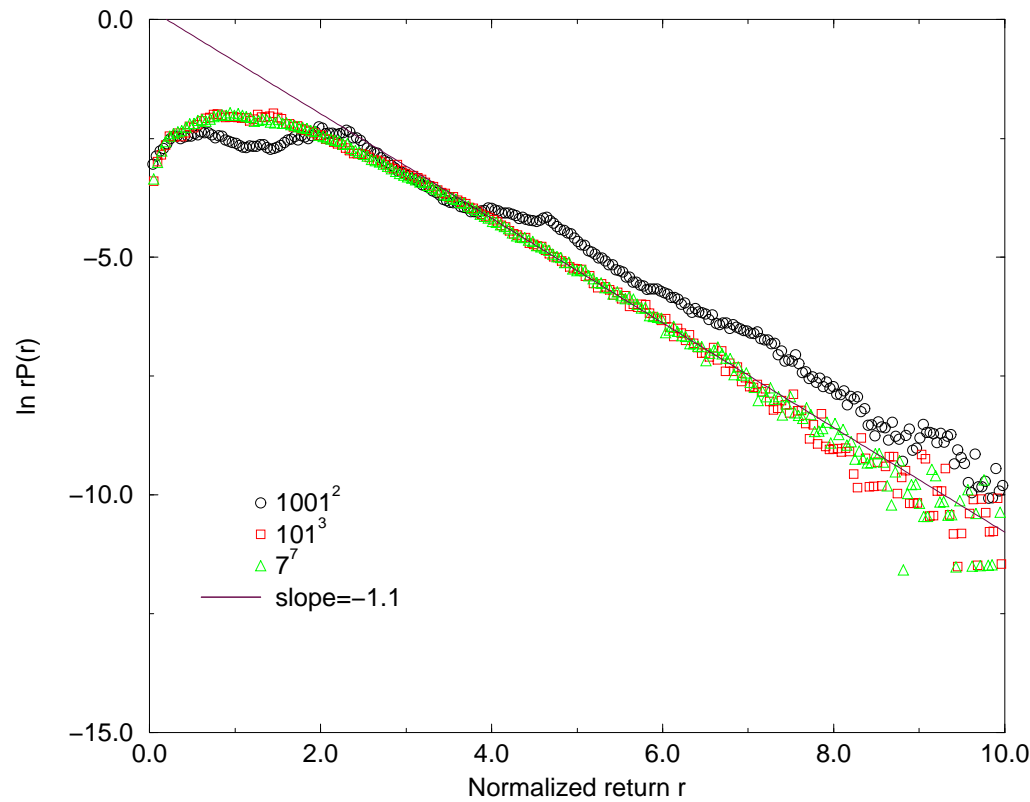

Fig. 5. Replot of Fig. 目 for $\ln r P(r)$ as a function of the normalized positive return $r$, with a straight line of slope -1.1 .

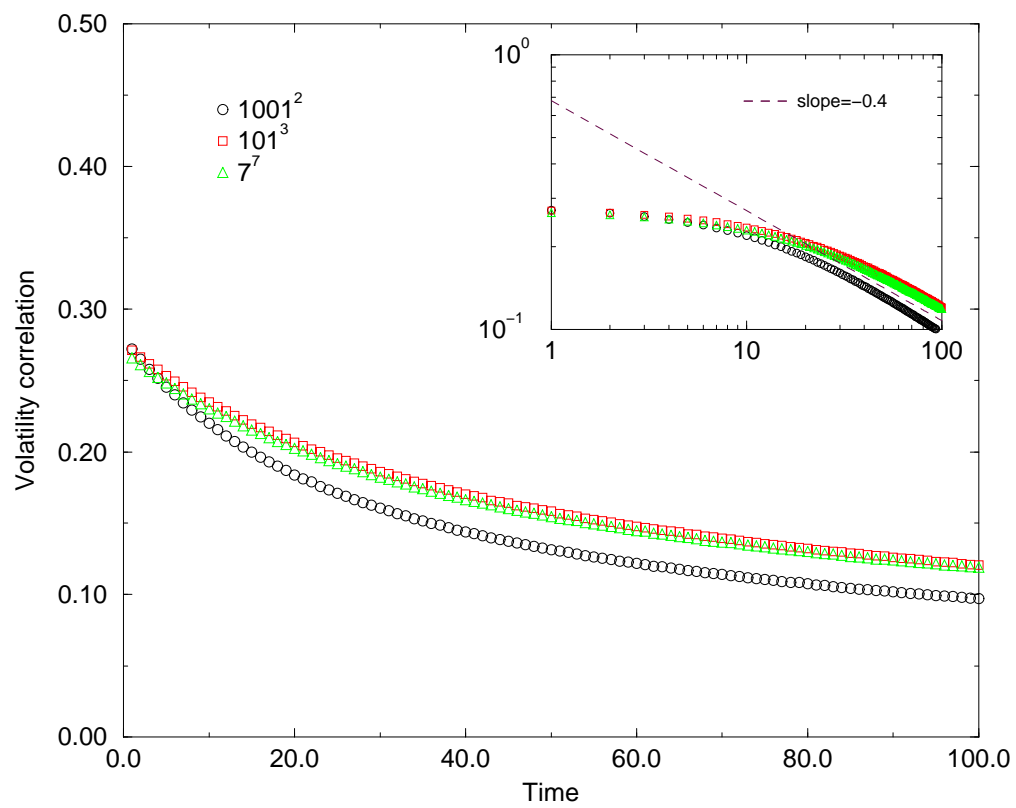

Fig. 6. Autocorrelations for the volatility in different simulations with the same parameters as Fig. A. The slow decay is displayed, and the inset gives the corresponding log-log plots which exhibit an asymptotic power-law behavior with an exponent about 0.4 for different systems. 


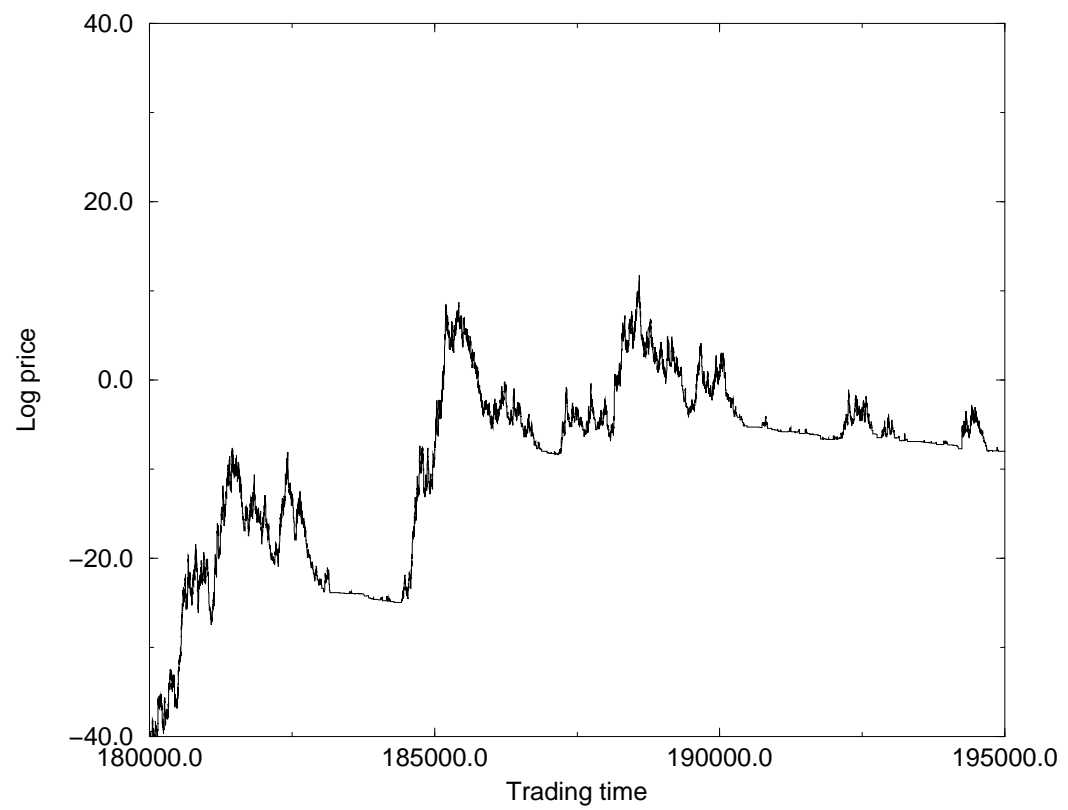

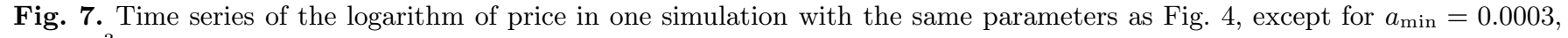
on a $31^{3}$ cubic lattice. 